\title{
Elektronisches Polleninformationsnetzwerk in Bayern (ePIN) und systematischer Literaturreview zu symptom-relevanten Konzentrationen von aerogenen Pollen (LuPo)
}

A Weber, H Mertes, N Steckling-Muschack, P Schutzmeier, S Kutzora, A Damialis, M Kabesch, C Traidl-Hoffmann, KC Bergmann, S Böse-O'Reilly, D Nowak, J Oteros, J Buters, C Herr, S Heinze

\section{Hintergrund:}

In Bayern wird derzeit vom Bayerischen Landesamt für Gesundheit und Lebensmittelsicherheit (LGL) in Kooperation mit dem Zentrum für Allergie und Umwelt der TU und Helmholtz Zentrum München ein elektronisches

Polleninformationsnetzwerk (ePIN) aufgebaut. Neben der Darstellung der aktuellen Pollenexposition aus ePIN auf der Webseite des LGL sollen zusätzlich evidenzbasierte Ergebnisse zu "symptom-relevanten Konzentrationen von aerogenen Pollen (LUPO)" erarbeitet werden.

\section{Methodik:}

Neue Technologien ermöglichen eine automatisierte Pollenerkennung. Statt manuell Daten aus der mikroskopischen Analyse einzuspeisen (Arbeitsweise konventioneller Pollenfallen), können diese in elektronischer Form und in kurzen zeitlichen Intervallen zur Verfügung gestellt werden. Geplant sind acht elektronische Pollenmonitore sowie vier manuelle Pollenfallen mit Schwerpunkt Klimaforschung und zur Detektion neuer Pollenarten. ePIN wird unterstützt von den ansässigen Gesundheitsämtern.

Im Projekt LUPO wird ein systematischer Literaturreview durchgeführt: bisherige Ergebnisse zu symptom-relevanten Konzentrationen von aerogenen Pollen werden systematisch recherchiert und unter Einbeziehung wissenschaftlich ausgewiesener Experten evaluiert. Basierend auf den Ergebnissen sollen Möglichkeiten und Grenzen zur Bewertung des Pollenflugs in Bezug auf die suszeptible Population dargestellt werden.

\section{Ergebnisse:}

Mit ePIN werden die Pollendaten schneller verfügbar sein. Die Bevölkerung kann sich auf der Webseite des LGL über den aktuellen Pollenflug informieren. Weiterhin soll ePIN die Datenlage für die allergie- und gesundheitsbezogene Klimaforschung sowie für das Klimamonitoring verbessern.

Im Projekt LUPO deckte die Kombination der Suchbegriffe die Beschreibung von Exposition, Grenzwert, Assoziation und gesundheitliches Outcome ab und hat 498 PubMed gelistete Treffer generiert. Von diesen konnten 20 Publikationen anhand des Titel- und Abstractscreenings eingeschlossen werden. Die in Zusammenhang mit der Pollenkonzentration untersuchten Outcomes sind hauptsächlich bronchiale, nasale und okulare Symptome sowie Krankenhauseinweisungen aufgrund von Asthmaexazerbationen. Es ist zu erwarten, dass sich die Anzahl projektrelevanter Studien nach Abschluss des Schneeballverfahrens und Volltextscreenings weiter erhöht.

\section{Ausblick:}

Die Erkenntnisse aus LUPO sollen für die Webseite des LGL zur Darstellung der Pollendaten aus ePIN genutzt werden um der interessierten Bevölkerung Zugang zu evidenzbasierten Forschungsergebnissen zum Zusammenhang zwischen Pollenflug und Symptomen zu ermöglichen. Zusätzlich werden im Projekt LIMA bisherige Erkenntnisse zu Maßnahmen zur Minderung von aeroallergen bedingten allergischen Beschwerden systematisch recherchiert.

ePIN: Finanziert durch das Bayerische Staatsministerium für Gesundheit und Pflege und durch das Bayerische Staatsministerium für Umwelt und Verbraucherschutz.

LUPO: Finanziert durch das Bayerische Staatsministerium für Gesundheit und Pflege 